\title{
Activité lipolytique de quelques micro-organismes
}

\section{II. - MOISISSURES}

\author{
par
}

\author{
R. ALIFAX
}

Laboratoire de Recherches de l'I.N.R.A.

Chaire des Industries Agricoles et Alimentaires de l'I.N.A. Paris-Grignon, 16, rue Claude-Bernard, Paris $5^{\mathrm{e}}$

\section{SOMMAIRE}

Ce travail constitue la seconde partie de notre étude sur l'activité lipolytique des micro-organismes (Alifax R. [1]).

Nous avons étudié, après une sélection, 13 souches de moisissures. Les acides gras libérés après hydrolyse des triglycérides ont été analysés qualitativement par chromatographie en phase gazeuse avec programmation de température.

Les propriétés lipolytiques de ces souches nous ont permis de les classer sommairement en trois groupes, selon leur aptitude à libérer qualitativement les acides gras à courtes chaînes :

$1^{\circ}$ Celles qui hydrolysent les triglycérides du beurre avec libération négligeable des acides gras à courtes chaînes.

2. Celles qui en libèrent peu.

$3^{\circ}$ Celles qui hydrolysent ces mêmes triglycérides avec libération notable d'acides gras à courtes chaînes.

\section{INTRODUCTION}

Les corps gras, s'ils se trouvent en contact avec des moisissures, peuvent être l'objet d'altérations de diverses natures. Quand il s'agit d'espèces lipolytiques, ces altérations résultent d'abord de processus d'hydrolyse, suivis ou non de réactions oxydatives. Ultérieurement, d'autres phénomènes peuvent conduire à la formation de produits nouveaux qui dérivent eux-mêmes des produits des réactions d'hydrolyse ou d'oxydation. Toutes ces transformations peuvent donner naissance à des produits néfastes ou désirables, affectant le goût, l'arôme et même l'aspect de la substance envisagée. Sous 
l'action des lipases, l'hydrolyse des triglycérides libère des acides gras. L'altération qui en résulte est la rancidité. Ultérieurement, si les moisissures possèdent dans leur équipement enzymatique une lipoxygénase, cette enzyme peut catalyser la fixation de l'oxygène de l'air sur des liaisons éthyléniques des acides gras insaturés. C'est l'altération appelée suiffage qui résulte de la formation de peroxydes et de dérivés cétoniques et aldéhydiques. Le processus de ces transformations peut être résumé dans le schéma suivant :

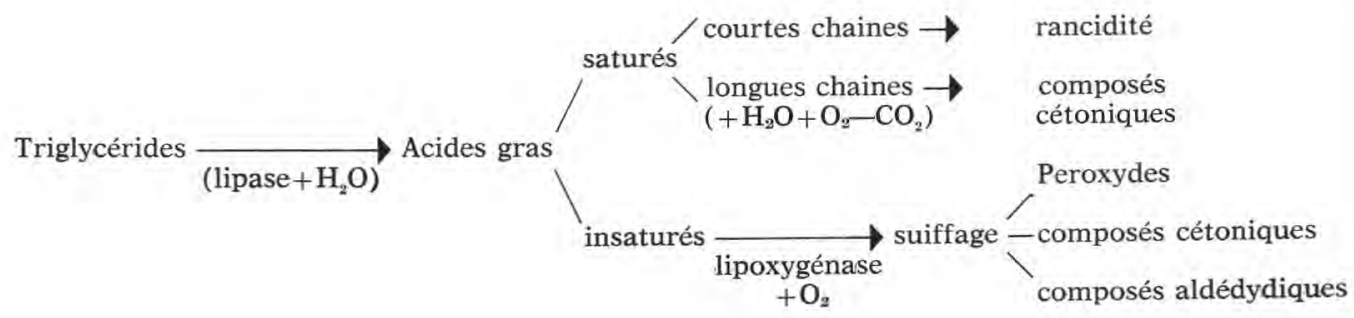

Les huiles d'origine végétale, malgré leur nature anhydre, peuvent aussi être altérées par les micro-organismes.

Ainsi Starkle (M.) [8] a isolé d'une huile rance Aspergillus niger et Aspergillus fumigatus. L'auteur a montré que ces moisissures, quand elles sont cultivées sur des acides gras comme seule source de carbone, en culture pure, produisent des méthylcétones. Le même auteur pense que la rancidité n'est pas due aux esters de l'acide butyrique, mais aux méthyl-propyl cétone et heptyl-méthyl cétone. Ces produits dériveraient de l'acide caproïque et de l'acide laurique avec perte d'un atome de carbone.

Il n'en est pas de même quand on s'adresse à des corps gras plus complexes, d'origine animale ou végétale. Le beurre et la margarine sont des émulsions. Ils comprennent une phase lipidique et une phase aqueuse. Cette dernière peut contenir, dans l'un ou l'autre cas, des traces de lait écrémé (lactose, acide lactique, protéines) ainsi que de l'amidon. Les micro-organismes, et notamment les moisissures, y trouvent, en raison de la présence de cette phase aqueuse, un substrat propice à leur prolifération. Etant aérophiles, elles peuvent former au contact de l'air, à la surface des produits, des taches ou un feutrage de couleurs variées qui les écartent de l'alimentation humaine et les relèguent au rang de matières premières pour la fabrication des aliments du bétail, avec les risques que cela comporte. Ainsi Pomeranz et Moreau, cités par Cognerai-Devillers L. [3], admettent que la rancidité cétonique conduit à des métabolites susceptibles de provoquer chez le porc des distrophies musculaires.

Enfin dans le cas d'altérations prononcées, le beurre et la margarine deviennent impropres à la consommation humaine ou 
animale et ne peuvent être utilisés que par l'industrie de la stéarinerie.

Dans un tout autre ordre d'idée, la détérioration des couches de vernis ou de peinture peut être, parfois, attribuée également à la présence de moisissures lipolytiques, comme l'ont souligné Rothwell F. M. [6] et Dresher R. F. [4].

Ces auteurs ont montré que Cladosporium sp., Pullularia pullulans, Alternaria sphaerospermum peuvent, dans certains cas, être partiellement ou totalement responsables des altérations des couches de vernis ou de peintures. Leur mycélium peut, en effet, à la faveur de l'humidité, s'insinuer dans les couches protectrices et provoquer, par leurs enzymes, des modifications dans la composition des huiles qui entrent dans leur préparation.

Richard T. Ross [5] a étudié plus particulièrement l'altération, par les micro-organismes, de l'huile de lin et des pellicules dans la composition desquelles elle intervient. L'auteur a montré que des moisissures du genre Cladosporium, Alternaria, Phoma et Pullularia possèdent une activité lipolytique spécifique pour les liaisons esters des triglycérides de l'huile de lin et une activité oxydative qui catalyse à la fois l'oxydation de la glycérine et celle des acides gras.

Ces informations nous ont conduit à étudier et à comparer l'activité lipolytique de quelques moisissures vis-à-vis de la matière grasse pure de beurre, comme nous l'avons fait, dans un précédent travail, avec des bactéries lipolytiques.

\section{PROTOCOLE EXPERIMENTAL}

\section{$1^{\circ}$ Choix et origine des souches}

Dans une première étape, par des essais rapides, nous avons sélectionné les souches présentant une propriété lipolytique accusée en appréciant simplement, dans le temps, le virage d'un indicateur, consécutif à la libération des acides gras et l'intensité de la coloration développée. Nous avons ainsi retenu 13 souches appartenant à 13 espèces différentes.

Parmi elles, certaines sont des souches de collection, appartenant aux espèces Penicillium roquefortii, Mucor racemosus, Mucor pusillus, Candida lipolytica, Candida paralipolytica. Les autres ont été isolées du sol et identifiées par le Laboratoire de Cryptogamie du Muséum d'Histoire Naturelle de Paris : Mucor circinellö̈des, Aspergillus fumigatus, Scopulariopsis brevicaulis, Fusarium poae, Fusarium oxysporium, Penicillium lanogriseum, Penicillium commune, Cladosporium. 


\section{$2^{\circ} \quad$ Ensemencement et récolte. Extraction des acides gras}

Ces opérations ont été conduites selon les techniques décrites dans notre étude sur l'activité lipolytique des bactéries.

Toutefois, les ensemencements ont été effectués à partir d'une suspension de spores pour chaque souche de moisissure. L'incubation des boîtes a été réalisée à $25^{\circ} \mathrm{C}$ pendant $5 \mathrm{j}$.

\section{$3^{\circ}$ Estérification des acides gras}

Les acides gras ont été estérifiés par butylation. Ce mode d'estérification a été préféré à la méthylation, dans le souci d'obtenir une meilleure séparation des acides gras à courtes chaînes. En effet, dans le travail précédent, la méthylation ne nous a pas permis de distinguer nettement les acides gras mineurs. En revanche, leur identification est facilitée par la butylation. Dans ce but, nous avons retenu la méthode décrite par Bézard J. et Bugaud M. [2].

\section{$4^{\circ}$ Chromatographie}

Appareil type Girdel.

Colonne $\left\{\begin{array}{l}\text { métal inox } \\ \text { longueur } 3 \mathrm{~m} \\ \text { diamètre } 1 / 8 \text { de pouce } \\ \text { phase stationnaire DEGS } 4 \text { p. } 100 \\ \text { support chromosorte G } 80-100 \text { mesh. }\end{array}\right.$

Détecteur : ionisation de flamme.

Gaz vecteur $\mathrm{N}^{2}$ : débit pression 1,5 bars.

Débit $\mathrm{H}^{2}: 30 \mathrm{ml} / \mathrm{mn}$.

Atténuation électromètre 512.

Température programmée : 80 à $200^{\circ} \mathrm{C}$.

\section{RESULTATS}

L'examen de l'ensemble des résultats nous a montré que les propriétés lipolytiques des souches étudiées permettaient de les classer sommairement en trois catégories. Nous reproduisons les trois profils chromatographiques qui illustrent ce classement.

Le profil I traduit l'activité lipolytique des moisissures dont les lipases ne libèrent pas d'acides gras à courtes chaînes (genre Mucor racemosus).

Le profil II traduit l'activité lipolytique des germes dont les lipases libèrent peu d'acides gras à courtes chaînes (genre Fusarium poae). 

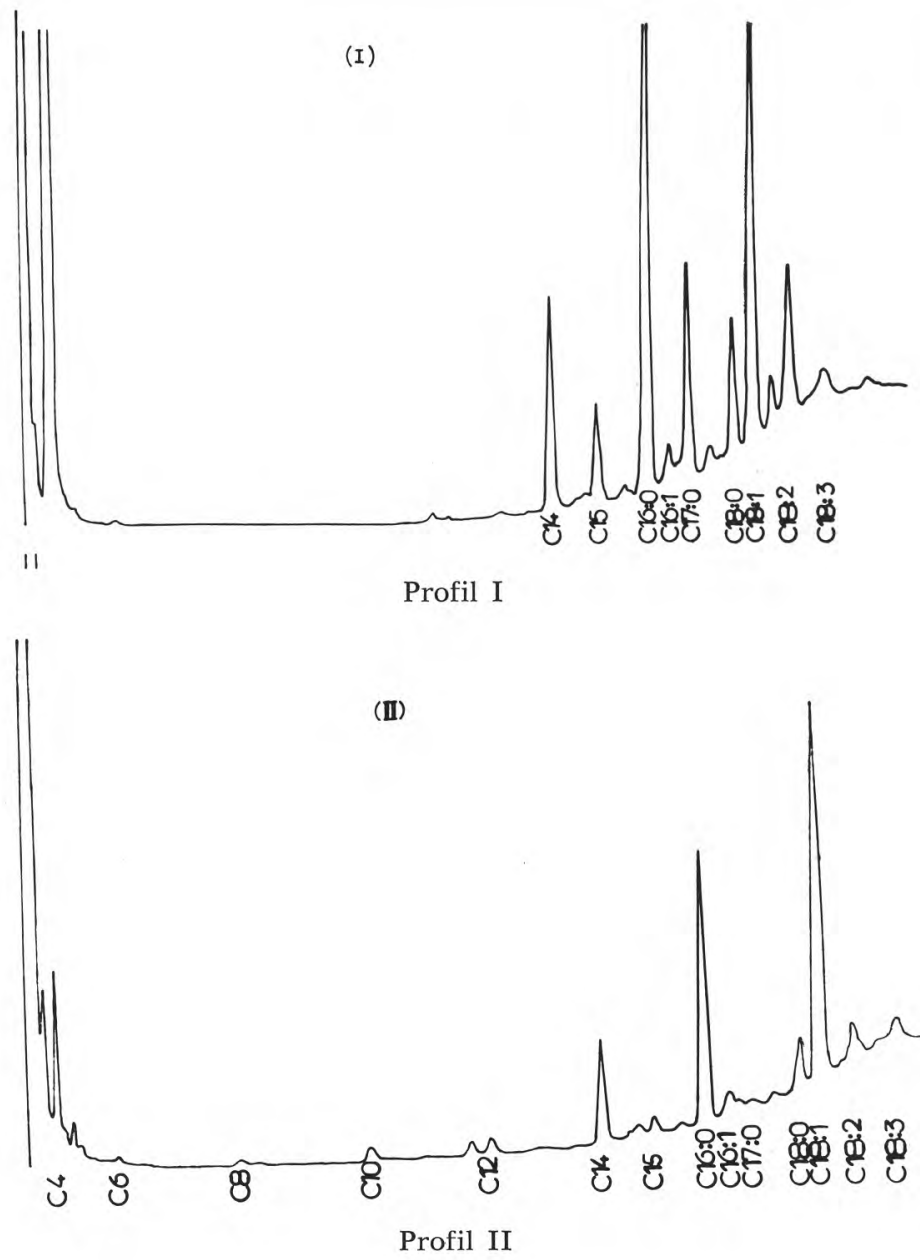

Enfin le profil III traduit l'activité lipolytique des moisissures dont les lipases hydrolysent les triglycérides avec libération appréciable d'acides gras à courtes chaînes (genre Penicillium roquefortii).

En ce qui concerne les acides gras supérieurs, nous notons une aptitude sensiblement égale à libérer l'acide palmitique et l'acide oléique. Les autres acides gras le sont dans des proportions très variées.

Ce classement sommaire confirme, dans son ensemble, les conclusions de Ruth Y. Mayne [7] tirées de son étude sur l'activité de divers micro-organismes, bactéries et moisissures, vis-à-vis de l'huile de coton. Selon leur activité lipolytique, l'auteur les classe en cinq 


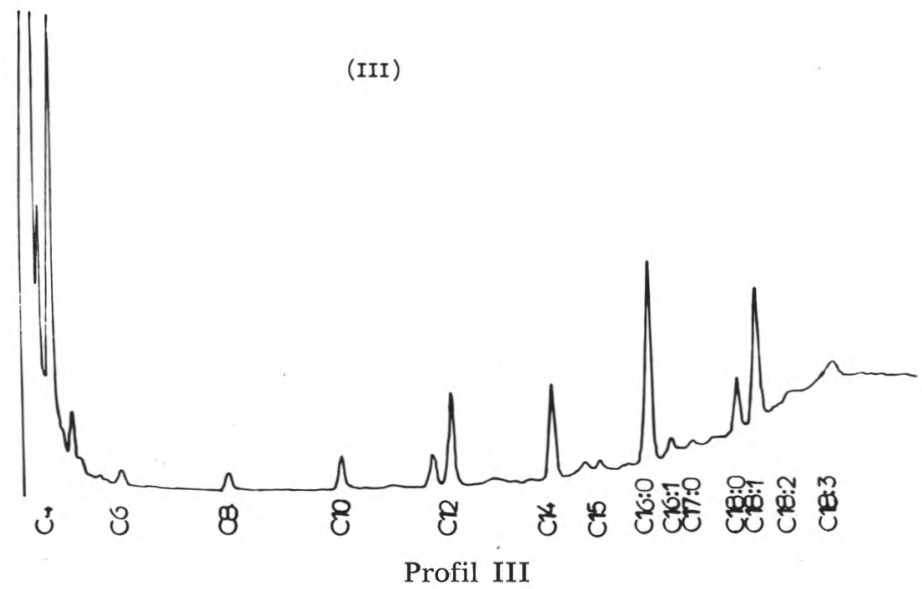

(IV - Témoin)

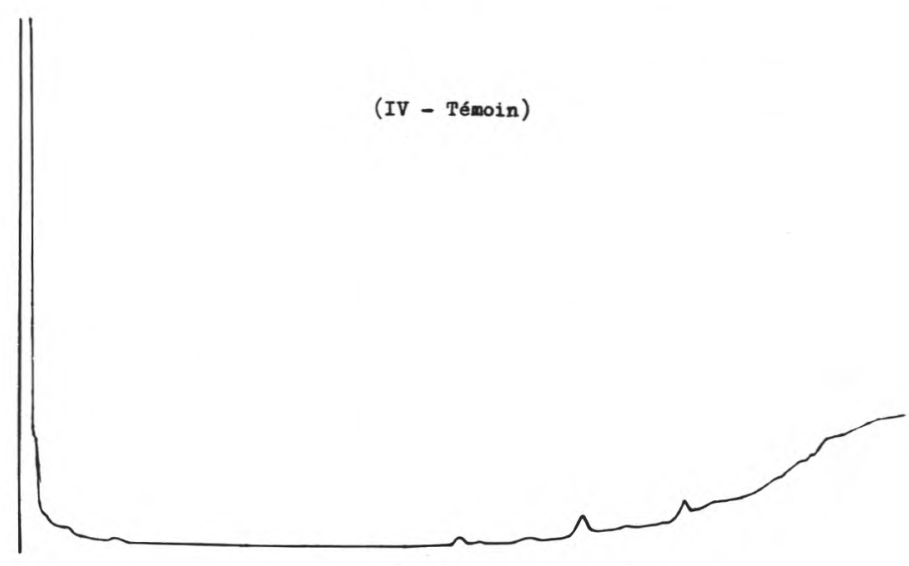

Profil IV (témoin)

groupes. Aspergillus flavus, Aspergillus awamori et Penicillium lanosum se situent dans les groupes II et III ; ils sont fortement lipolytiques avec accumulation modérée ou faible d'acide gras. Dans le groupe V, l'auteur retient cinq moisissures du genre Fusarium, dont $F$. oxysporium, qu'il considère comme modérément lipolytiques.

Nous reproduisons, avec un profil témoin, les tracés chromatographiques qui traduisent les propriétés lipolytiques qualitatives de l'ensemble des souches que nous avons examinées. Le profil témoin est obtenu à partir du même échantillon de matière grasse traité dans les mêmes conditions.

Le tableau 1 traduit ces résultats. 


\section{TABLEAU 1}

Représentation qualitative des acides gras libérés des triglycérides du beurre par trois moisissures lipolytiques

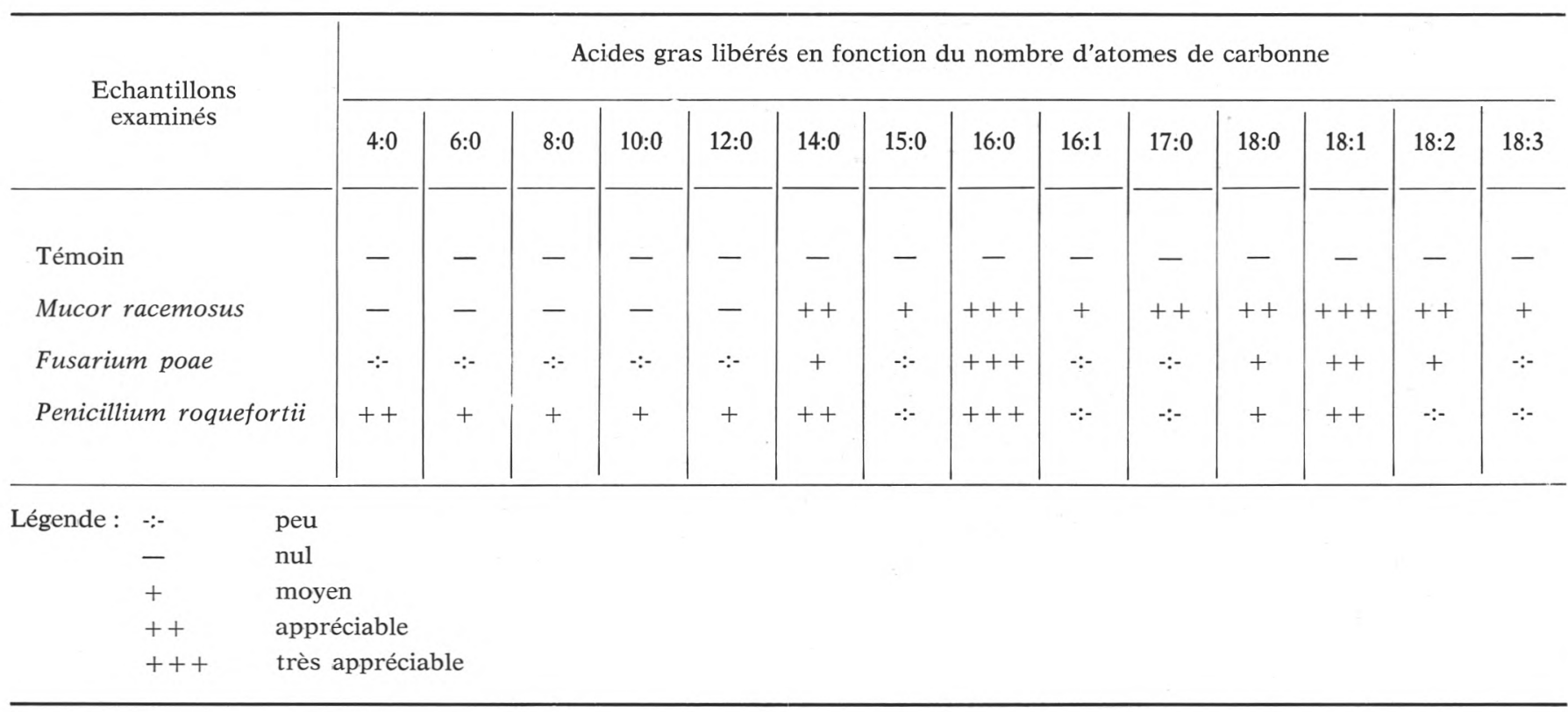


Ci-dessous : photographie montrant la réaction de lipolyse.

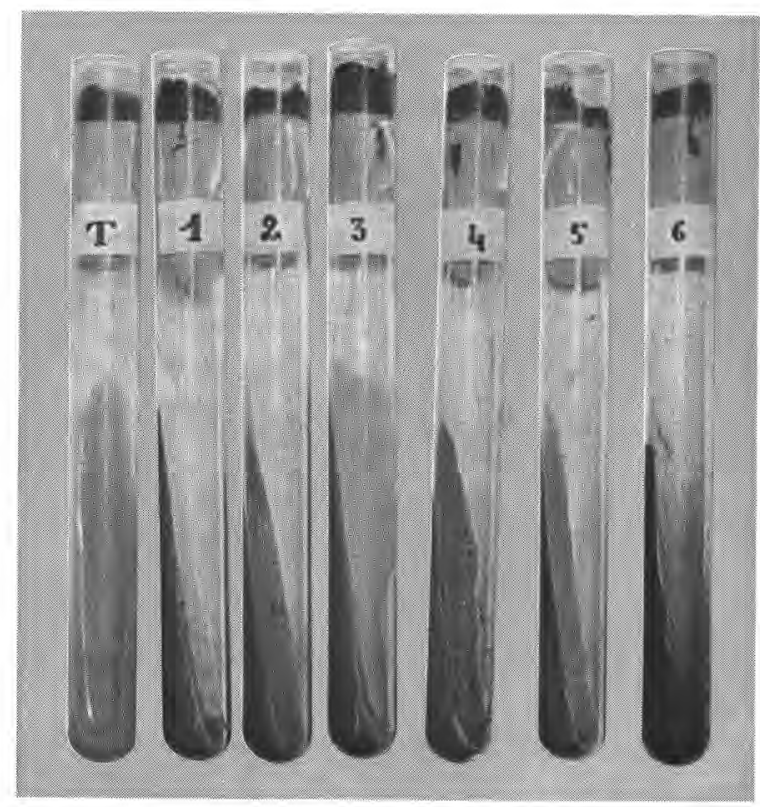

Substrat : matière grasse du beurre.

Indicateur de réaction : Bleu Victoria.

T: Témoin culture.

1: Fusarium poae.

2: Penicillium lanogriseum.

3: Mucor racemosus.

4: Penicillium roquefortii.

5 : Scopulariopsis brevicaulis.

6: Cladosporium.

\section{DISCUSSION}

La facilité avec laquelle leurs spores peuvent être disséminées fait que les moisissures constituent de redoutables agents de contamination. Toutes les denrées alimentaires sont donc exposées à leur action. Quand il s'agit de corps gras contaminés par des moisissures lipolytiques, le degré de lipolyse des triglycérides peut être très important si leurs enzymes sont exocellulaires et le développement mycélien surtout abondant.

Outre la libération des acides gras, il peut y avoir formation de produits secondaires sur la nature desquels nous possédons peu de 
renseignements. Leur identité et l'étude de leurs propriétés compléteraient utilement nos connaissances sur leur rôle bénéfique ou néfaste dans les modifications de flaveur présentées par les denrées alimentaires au cours de leur conservation.

La réunion de ces informations serait également de nature à mieux orienter la sélection des souches en vue de la production d'enzymes microbiennes utilisables éventuellement dans certaines technologies industrielles.

$\mathrm{Au}$ cours de ce travail, nous avons limité nos observations à l'examen du comportement des triglycérides de la matière grasse du beurre. Nous pensons que l'extension de l'étude à d'autre triglycérides, ceux des huiles végétales notamment, mérite une attention toute particulière. En effet, leur composition varie d'une plante à l'autre. Les huiles constituent donc des substrats de choix, susceptibles de nous renseigner sur le mode d'action ainsi que sur la spécificité des lipases d'origine fongique vis-à-vis de ces différents triglycérides.

Nos travaux sur la lipolyse sont actuellement orientés dans cette voie.

\section{R é s u m é}

En utilisant comme substrat la matière grasse pure du beurre. nous avons étudié les propriétés lipolytiques de quelques moisissures provenant de collection ou isolées du sol. Leurs propriétés lipolytiques nous ont permis de les classer qualitativement en trois groupes qui se distinguent notamment par l'importance plus ou moins grande de la libération des acides gras à chaînes courtes.

\section{S u $\mathrm{m}$ m a r y}

By using the pure butter fat as a substratum, we have studied the lipolytic properties of some molds, from collection or isolated from soil. Their lipolytic properties have permitted their classification in three groups distinguished by their ability to free short fatty acids from triglycerids of butter.

\section{Zusammenfassung}

Mit dem reinen Fettstoff des Butters als Substrat haben wir die lipolytischen Fähigkeiten einiger Schimmel entweder von einer Kollection aus oder vom Grund aus isoliert untersucht. Ihre 
lipolytischen Fähigkeiten haben uns erlaubt, sie in drei Gruppen zu klassifieren, welche sich hauptsätzlich bei der verschieden bedeutenden Erzeugung von kurzkettigen Fettsäuren voneinander unterscheiden lassen.

\section{Remerciements}

Nous adressons nos sincères remerciements à $M$. le professeur Fusey du Laboratoire de Cryptogamie du Muséum d'Histoire Naturelle de Paris, qui a bien voulu procéder à l'identification des souches de moisissures isolées du sol.

Nos remerciements s'adressent également à $M$. le professeur Bézard, de la Faculté des Sciences de Dijon, Chaire de Physiologie animale, ainsi qu'à ses collaborateurs, qui ont analysé, par chromatographie en phase gazeuse, les acides gras libérés par les lipases des différentes souches de moisissures. Nous avons bénéficié de leur expérience pour l'interprétation des différents profils chromatographiques.

\section{Bibliographie}

[1] AlifaX (R.) (1972). - Activité lipolytique de quelques micro-organismes. I. Bactéries. Le Lait, mai-juin, 515-516, 283-296.

[2] Bezard (J.) and Bugaud (M.) (1972). - The componement triglycerides of rate adipose tissue. I. As studied after fractionnation into classes by silver ion Tin layer chromatography. Journal of Chromatography, 10, $451-462$.

[3] Coignerai-Devillers (L.) (1969). - Hygiène et bactériologie des margarines. Revue Française des Corps Gras, 8-9, 561-571.

[4] DRESHER (R. F.) (1958). - Microbiology of Paint Films. IV. Isolation and identification of the microflora of exterior emulsion paint. Ann. Paint. J., 42 (27). 80-102.

[5] RichaRd (T.) Ross (1958). - Microbiology of Paint Films. III. Attack of linseed oil and linseed oil films by microorganisms. Official Digest Federation Paint \& Varnish Prod. Clubs, 39, 377-391.

[6] Rothwell (F. M.) (1958). - Microbiology of Paint Films. II. Isolation and Identification of microflora on exterior oil paints. Official Digest Federation Paint and Varnish Prod. Clubs, 39, 368-376.

[7] Ruth (Y.) MAYNE (1956). - Lipolytic Activity of various microorganisms isolated from cotton seed. Applied Microbiology, 4, 270-273.

[8] Starkle (M.) (1924). - Die Methylketone im oxydativen Abbau einiger Triglyceride (bzw. Fettsauren) durch SchimmelPilze unter Berücksichtigung der besonderen Ranzidität des Kolosfettes. I. Die Bedeutung der Methylketon im Biochemismus der ButterRanzidität. II. Uber die Entstehung und Bedeutung der Methylketone als Aromastoffe im RoquefortKäse. Biochemische Zeitschrift., 151. 